\title{
Intermediate Volume on Computed Tomography Imaging Defines a Fibrotic Compartment that Predicts Glomerular Filtration Rate Decline in Autosomal Dominant Polycystic Kidney Disease Patients
}

\author{
Anna Caroli, ${ }^{\star \dagger}$ Luca Antiga, ${ }^{\star}$ Sara Conti, ${ }^{,}$ \\ Aurelio Sonzogni, ${ }^{\S}$ Giorgio Fasolini," \\ Patrizia Ondei, \\ Giuseppe Remuzzi, ${ }^{\| * *}$ and Andrea Remuzzi*†† \\ From the Departments of Biomedical Engineering," Molecular \\ Medicine, ${ }^{\neq}$and Renal Medicine, ${ }^{* *}$ Mario Negri Institute for \\ Pharmacological Research, Bergamo; the Laboratory of \\ Epidemiology, Neuroimaging and Telemedicine, ${ }^{\dagger}$ IRCCS \\ Fatebenefratelli, Brescia; the Units of Pathology, Radiology, "I and \\ Nephrology and Dialysis," Azienda Ospedaliera Ospedali Riuniti \\ di Bergamo, Bergamo; and the Department of Industrial \\ Engineering, ${ }^{\text {t+ }}$ University of Bergamo, Bergamo, Italy
}

Total kidney and cyst volumes have been used to quantify disease progression in autosomal dominant polycystic kidney disease (ADPKD), but a causal relationship with progression to renal failure has not been demonstrated. Advanced image processing recently allowed to quantify extracystic tissue, and to identify an additional tissue component named "intermediate," appearing hypoenhanced on contrastenhanced computed tomography (CT). The aim of this study is to provide a histological characterization of intermediate volume, investigate its relation with renal function, and provide preliminary evidence of its role in long-term prediction of functional loss. Three ADPKD patients underwent contrast-enhanced CT scans before nephrectomy. Histological samples of intermediate volume were drawn from the excised kidneys, and stained with hematoxylin and eosin and with saturated picrosirius solution for histological analysis. Intermediate volume showed major structural changes, characterized by tubular dilation and atrophy, microcysts, inflammatory cell infiltrate, vascular sclerosis, and extended peritubular interstitial fibrosis. A significant correlation $(r=-0.69, P<$ 0.001) between relative intermediate volume and baseline renal function was found in 21 ADPKD patients. Long-term prediction of renal functional loss was investigated in an independent cohort of 13 ADPKD patients, followed for 3 to 8 years. Intermediate volume, but not total kidney or cyst volume, significantly correlated with glomerular filtration rate decline $(r=-0.79, P<0.005)$. These findings suggest that intermediate volume may represent a suitable surrogate marker of ADPKD progression and a novel therapeutic target. (Am J Pathol 2011, 179:619-627; DOI: 10.1016/j.ajpath.2011.04.036)

Autosomal dominant polycystic kidney disease (ADPKD) is the most common renal hereditary disorder and the fourth leading cause of end-stage renal disease (ESRD) in adults. ${ }^{1,2}$ ADPKD can arise from mutations in either the $P K D 1$ gene (which encodes the protein polycystin 1) or the PKD2 gene (encoding polycystin 2). PKD1 is more severe than $P K D 2$-associated disease, the mean age of onset of ESRD being 54 years and 74 years for PKD1 and PKD2, respectively. ${ }^{3}$

The clinical hallmark phenotype of this inherited condition is the progressive and marked enlargement of the kidneys caused by sustained expansion of multiple fluidfilled cysts originating from the tubule wall ${ }^{4}$ leading to crowding of adjacent nephrons and to injury of normal parenchyma. $^{5-9}$ In ADPKD, renal function remains normal for a long period of time before starting to decline inexorably when the noncystic parenchyma has incurred serious damage. ${ }^{10}$ This often leaves patients who have normal renal function with the false belief that little renal

\footnotetext{
Supported in part by grant n. 01TRN07a from the PKD Foundation. Accepted for publication April 11, 2011.

Address reprint requests to Anna Caroli, Ph.D., Mario Negri Institute for Pharmacological Research, Via Camozzi 3, 24020, Ranica (BG), Italy. E-mail: acaroli@marionegri.it.
} 
damage has occurred. This points out the urgent need of reliable markers of disease progression other than glomerular filtration rate (GFR), capable of detecting existing damage and possibly predicting progression over a relatively short period of time while renal function, as measured by serum creatinine or estimated GFR (eGFR), is normal or relatively preserved. The identification of such markers is deemed necessary both for clinical follow-up and for testing new therapeutic strategies in the context of clinical trials.

Since renal enlargement due to cyst growth is the underlying macroscopic process, image-based measurements have been used in the literature to quantify kidney disease progression in ADPKD. ${ }^{11-14}$ The CRISP (Consortium for Radiological Imaging Studies of Polycystic Kidney Disease) study showed that in ADPKD patients, total kidney volume and cyst volume increase exponentially with age ${ }^{11}$ and that magnetic resonance imaging can reproducibly detect this change. ${ }^{8,12,13} \mathrm{How}$ ever, a causal relationship between kidney or cyst volume increase and the ultimate progression to renal failure has not been demonstrated. On the other hand, changes in noncystic renal tissue components, which may in principle be closely connected to GFR decline in ADPKD, are not necessarily directly dependent on changes in kidney or cyst volume. The increasing resolution of imaging systems now available allows one to reliably discern cystic and noncystic tissue components. ${ }^{15}$ In a recent study, ${ }^{15}$ we took advantage of advanced image processing techniques to characterize extracystic tissue on contrast-enhanced computed tomography (CT). In that work, we subdivided extracystic tissue into two separate components, fully enhanced parenchyma and hypoenhanced tissue, which we called "intermediate." ${ }^{15}$ We found that in patients with ADPKD and normal renal function to severe renal dysfunction, the ratio of intermediate volume relative to parenchymal volume significantly correlated with both baseline GFR and GFR changes over the 6-month follow-up period. ${ }^{15}$ In that small cohort of patients, neither cyst nor parenchyma volumes correlated with GFR and GFR decline. Thus, quantification of the noncystic fraction of the polycystic kidney may provide an effective image-based method to gauge renal function loss ${ }^{16}$ and predict the rate of decline more reliably than total kidney or total cyst volume. However, the nature of intermediate volume and its role in long-term renal function loss remain ill defined. Actually, there are a number of preclinical studies showing that diffuse interstitial fibrosis occurs within renal parenchyma in ADPKD. ${ }^{17-19}$ Moreover, the presence of interstitial fibrosis and tubular atrophy in patients with ADPKD and incipient renal failure has been documented in the context of a clinical study. ${ }^{20}$ The purpose of the present study was then to characterize the structural nature of intermediate tissue on kidneys excised from three ADPKD patients with end-stage renal disease already on hemodialysis who underwent contrast-enhanced CT before surgery, relating radiological findings with histological findings. In addition, we investigated the relation between intermediate volume and renal function. This was done in two independent cohorts of ADPKD patients, enrolled in two distinct studies, the
SIRENA (Sirolimus Treatment in Patients with Autosomal Dominant Polycystic Kidney Disease: Renal Efficacy and Safety) study ${ }^{21}$ aimed to assess the effects of mTOR inhibition on disease progression (SIRENA cohort) and the clinical study we performed ${ }^{22}$ to assess the effect of somatostatin treatment on disease progression (somatostatin cohort). Specifically, the SIRENA cohort data were used to further investigate the previously reported correlation between intermediate volume and GFR. ${ }^{15}$ The somatostatin cohort data, augmented by long-term clinical follow-up observations, were used to assess the power of intermediate volume to predict renal function decline over a 5-year follow-up.

\section{Materials and Methods}

\section{Patients}

Between October 2008 and September 2009, three consecutive ADPKD patients underwent bilateral nephrectomy at the Nephrology Unit of the Azienda Ospedaliera Ospedali Riuniti di Bergamo, after providing their written informed consent. Patient 1 (female, 67 years old) had polycystic kidney disease, known since the age of 29, with both kidney and liver involvement, and a family history of ADPKD (mother). At the age of 65, she developed ESRD and started renal replacement therapy with hemodialysis. A few months later, she had repeated episodes of sepsis secondary to cholelithiasis and/or infections of liver and kidney cysts. She underwent cholecystectomy and bilateral nephrectomy. Patient 2 (male, 38 years old) had polycystic kidney disease, known since the age of 17, and a family history of ADPKD (mother). Six months after starting hemodialysis due to ESRD (at the age of 35), he underwent binephrectomy due to repeated macrohematuria episodes in preparation for renal transplantation. Patient 3 (male, 48 years old) had polycystic kidney disease, known since birth, and a family history of ADPKD (brother). He started hemodialysis treatment at the age of 48 and, after 2 months, underwent binephrectomy due to recurrent episodes of abdominal pain that was thought to be due to his cystic kidneys. No complications arose during the nephrectomies in patients 1 and 2; patient 3 had significant bleeding, and the overall blood loss (about $700 \mathrm{~mL}$ ) was reintegrated. Before surgery, all patients underwent a contrast-enhanced CT scan.

\section{CT Acquisition}

CT acquisition was performed using a 64-slice CT scanner (LightSpeed VCT; GE Healthcare, Milwaukee, WI). A single breath-hold scan (120 kV; 150 to 500 mAs; matrix $512 \times 512$; collimation $2.5 \mathrm{~mm}$; slice pitch 0.984 ; increment $2.46 \mathrm{~mm}$ ) was initiated 80 seconds after injection of $100 \mathrm{~mL}$ (nominal dose, adjusted by patient weight) of an iodinated nonionic contrast agent (lomeron 350; Bracco, Italy) at the rate of $2 \mathrm{~mL} / \mathrm{second}$. Once acquired, images were transferred in DICOM 16-bit format from the clinical scanner onto PC workstations for subsequent processing. 
Intermediate Volume Identification on Late-Stage ADPKD CT Images

We previously developed an automatic procedure to identify and quantify intermediate volume ${ }^{15}$ based on the presence of three different renal tissue components (cyst, intermediate, and parenchymal volumes). To determine intermediate volume on late-stage ADPKD CT scans, where residual parenchyma is not detectable, we computed the intensity range of intermediate volume in the 21 ADPKD baseline CT scans of the SIRENA cohort (ADPKD patients with GFR $\geq 40 \mathrm{~mL} /$ minute), ${ }^{21}$ acquired using the same CT scanner and acquisition protocol as adopted in the present study. The minimum intermediate volume intensity in the previously acquired CT scans was $41 \pm 12$ Hounsfield Units (HU), whereas the maximum intensity was $91 \pm 23 \mathrm{HU}$. Intermediate volume intensity (in terms of $\mathrm{HU}$ ) was found to be independent of patient weight, in line with the fact that contrast agent dose was determined based on patient weight. No significant difference was found between mean intermediate volume intensity in patients with low $(<300 \mathrm{~mL})$ and high $(\geq 300$ $\mathrm{mL}$ ) parenchymal volume, suggesting that intermediate volume intensity does not depend on the amount of residual parenchyma. The average intermediate volume intensity range (41 to $91 \mathrm{HU}$ ) was restricted to (50 to 80 $\mathrm{HU})$ to exclude partial volume effects, and the latter range was used to identify intermediate volume in the three patients considered in the present study.

\section{Intermediate Tissue Identification on Excised Kidneys}

After nephrectomy, kidneys were fixed in formalin by immersion and stored at $4^{\circ} \mathrm{C}$ for 24 hours. To collect tissue samples from excised kidneys that corresponded to intermediate volume on CT scans, an image-guided harvesting procedure was set up. For each patient, kidney outlines were traced on CT images by a trained operator (A.C.), using an interactive image editing software (ImageJ; NIH, http://rsbweb.nih.gov/ij). Three-dimensional (3D) renderings of the outlined CT images were then generated using the 3D Slicer software (http:// www.slicer.org). At the time of sample collection, the 3D rendering, displayed on a laptop at the harvesting site, was rotated to match the orientation of the excised kidney on the bench. Kidneys were then transversally sectioned, and the corresponding reformatted CT slice was generated in the rendering software to guide kidney tissue harvesting. Tissue samples were then excised from kidneys under image guidance as shown in Figure 1. Samples from kidney tissue (approximately $1 \mathrm{~cm}^{3}$ ) located in areas of the reformatted image identified as intermediate on CT scans and from cyst walls were collected (Figure 1).

\section{Histological Characterization}

Kidney tissue samples corresponding to intermediate volume were processed for light microscopy using stan-
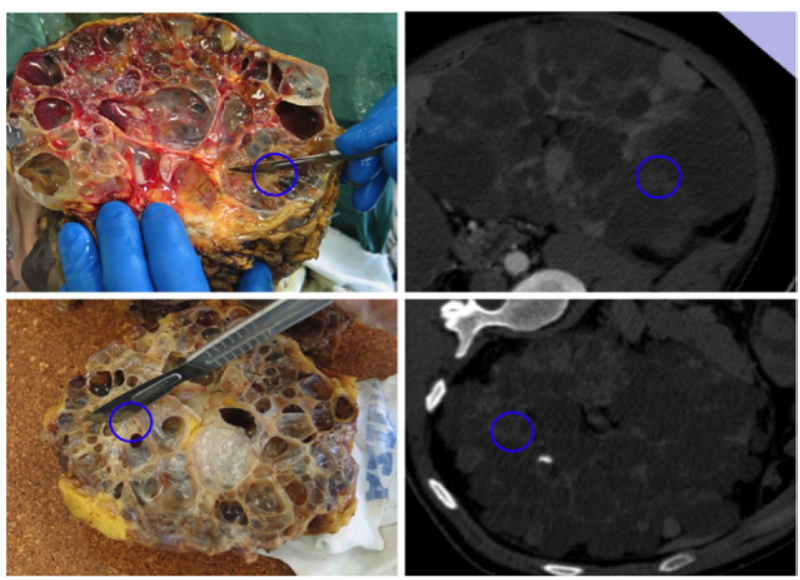

Figure 1. Collection of histological samples from the excised kidneys under guidance of 3D-reconstructed CT scans. Samples were drawn from tissue identified as intermediate on CT scans (top: left kidney from patient 2 , bottom: right kidney from patient 3). Blue circles highlight areas identified as intermediate on CT scans (right) and corresponding samples collected from the excised kidneys (left).

dard techniques. ${ }^{23}$ Each sample was post-fixed in $10 \%$ neutral formalin, dehydrated in alcohols, and then embedded in paraffin. Three-micron-thick sections (UItrotome V; LKB, Bromma, Sweden) were stained with $\mathrm{H} \& \mathrm{E}$ reagent for histological evaluation and with saturated picrosirius solution ( $0.1 \%$ Sirius Red in picric acid) for collagen staining. For Sirius Red staining, slides were taken through graded ethanol solution (100\%, 90\%, 80\%) into distilled water. Slides were then stained for 1 hour in saturated picric acid with $0.1 \%$ Sirius Red (Aldrich Chemical, Milwaukee, WI). After incubation with Sirius Red, slides were washed in $0.01 \mathrm{~N}$ hydrochloric acid for 2 minutes, rapidly dehydrated through graded ethanol solution and toluene, and finally mounted with a coverslip in Eukitt mounting medium ( $\mathrm{GmbH}$, Freiburg, Germany). Descriptive morphological evaluation was performed for the different tissue components, namely glomerular, tubular, and vascular structures, as well as renal interstitium, including matrix deposition and cell infiltrates. As control condition, normal renal tissue from kidney sections excised because of adenocarcinoma was processed and evaluated in parallel.

Renal infiltrating macrophages were identified by immunoperoxidase staining with an antibody directed against a specific monocyte/macrophage antigen, CD68 (mouse anti-human macrophages; DakoCytomation, Glostrup, Denmark). The paraffin-embedded kidney sections $(3 \mathrm{~mm})$ were deparaffinized and rehydrated. For immunoperoxidase analysis of CD68 antigen, the sections were incubated for 30 minutes with $0.3 \% \mathrm{H}_{2} \mathrm{O}_{2}$ in methanol to quench endogenous peroxidase and permeabilized in $0.1 \%$ Triton $\mathrm{X}-100$ in PBS $0.01 \mathrm{~mol} / \mathrm{L}(\mathrm{pH} 7.2)$ for 30 minutes. Before quenching endogenous peroxidase, kidney samples were treated with proteinase-K (20 $\mathrm{mg} / \mathrm{mL}$; Sigma-Aldrich, Milan, Italy) for 10 minutes at $37^{\circ} \mathrm{C}$, instead of Triton $\times 100$, followed by microwave [twice for 5 minutes in citrate buffer for $10 \mathrm{mmol} / \mathrm{L}(\mathrm{pH} 6)$ at an operating frequency of $2450 \mathrm{MHz}$ and $600-\mathrm{W}$ power output] and citrate buffer (15 minutes) incubations. Pri- 
mary antibody was diluted (1:100) and added overnight at $4^{\circ} \mathrm{C}$. Subsequent steps included incubations with the secondary biotinylated antibody [sheep anti-mouse IgC (Chemicon International, Temecula, CA)], avidin-biotin peroxidase complex solution, and finally, development with diaminobenzidine. The sections then were counterstained with Harris hematoxylin (Biooptica, Milan, Italy). Negative controls were obtained by omitting the primary antibody on adjacent sections.

\section{Correlation between Intermediate Volume and Renal Function}

We retrospectively considered the 21 ADPKD patients of the SIRENA cohort. ${ }^{21}$ Using the same quantification method previously described, ${ }^{15}$ we computed Pearson's correlation between the volume of each tissue component, including relative intermediate volume (defined as the ratio of intermediate volume over residual parenchymal volume, as described in our previous work ${ }^{15}$ ) and GFR, determined by iohexol plasma clearance. ${ }^{24}$ To further validate the relationship between intermediate volume and renal function at the time of CT scan, we also pooled the data from the SIRENA cohort with data from the somatostatin cohort, on which this correlation had been first identified. ${ }^{15}$

\section{Long-Term Prediction of Renal Function Decline by Intermediate Volume}

To investigate the potential long-term predictive power of intermediate volume on renal function decline, we evaluated the follow-up of the somatostatin cohort ${ }^{22}$ from 2001 to 2010. Long-term GFR decline in individual patients was estimated using longitudinal data available in electronic outpatient medical records at the Nephrology Unit of Azienda Ospedaliera Ospedali Riuniti di Bergamo. GFR estimation was performed for each clinical observation using plasma creatinine and the abbreviated MDRD (Modification of Diet in Renal Disease) formula (four variables). ${ }^{25}$ The individual slope of GFR decline (ie, the slope of the regression line of eGFR versus observation time) was computed. Because some patients started dialysis treatment during the follow up period, only predialysis follow-up data were considered. Patients with observation times shorter than 30 months or eGFR lower than $30 \mathrm{~mL} /$ minute $/ 1.73 \mathrm{~m}^{2}$ at baseline were excluded from the analysis. Thus, these patients had normal renal function or moderate renal insufficiency at the time of CT imaging. The relationship between relative intermediate volume at the time of CT acquisition and slope of GFR decline ( $\mathrm{mL} /$ minute/month) over the observation period was then evaluated. The same analysis was repeated to investigate the relationship between either total kidney or cyst volume and the slope of GFR decline.

\section{Statistical Analyses}

The relationship between intermediate volume and renal function was evaluated by Pearson's correlation. Individ-
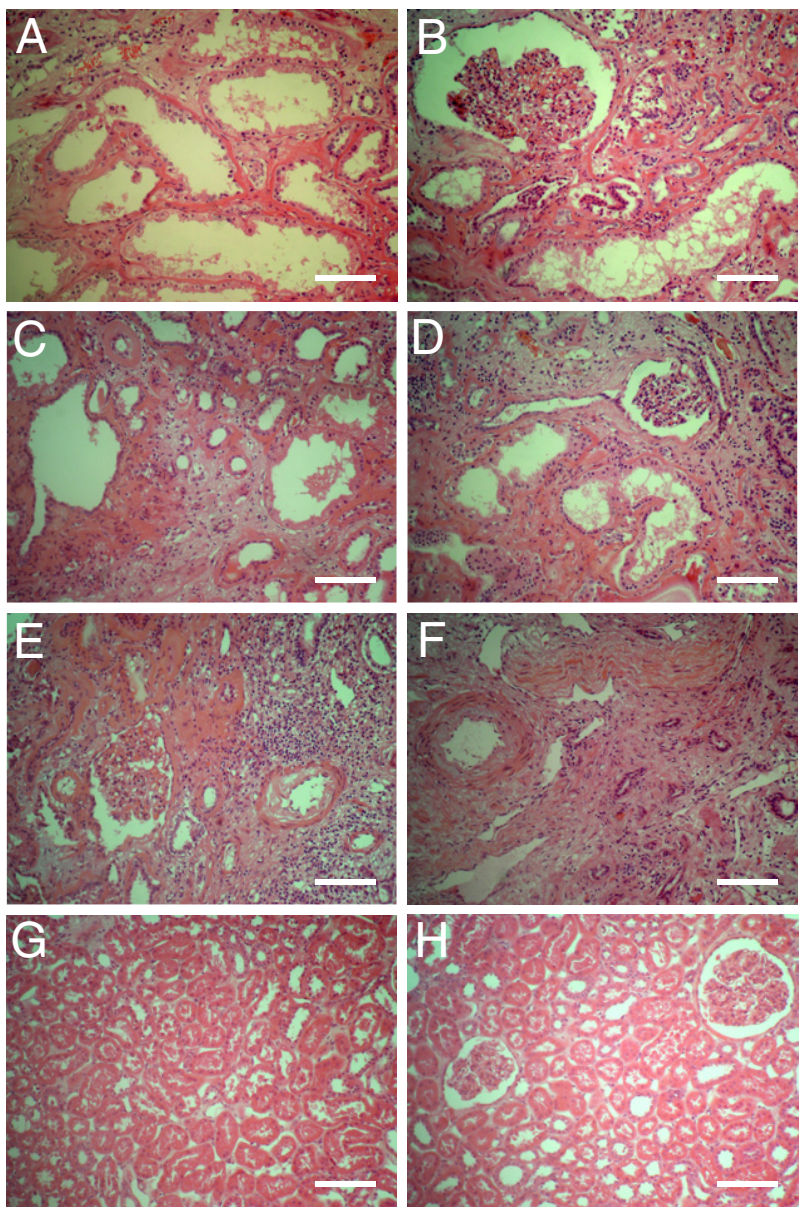

Figure 2. Representative images of the morphological analysis performed on kidney sections, in a patient with polycystic disease $(\mathbf{A}-\mathbf{F})$ and in a patient with normal-structure kidneys $(\mathbf{G}$ and $\mathbf{H})$, stained by H\&E. Scale bar $=100$ $\mu \mathrm{m}$

ual GFR decline was computed by linear regression of eGFR versus observation time. The relationships between intermediate volume, total kidney or cyst volume, and regression slopes were evaluated by Pearson's correlation. All statistical analyses were performed using the R statistical software (http://www.r-project.org)

\section{Results}

\section{Histological Characterization}

Renal samples for histological characterization of intermediate volume were collected from tissue areas matching the image identified as intermediate volume on CT scans. Representative images of the histological analysis performed on tissue sections stained with H\&E and Sirius Red are reported in Figures 2 and 3, respectively. In all ADPKD samples $(A-F)$, the renal parenchyma structure was severely altered as compared to normal kidney tissue $(\mathrm{G}$ and $\mathrm{H})$. Tubular dilatation and microcyst are the major changes documented (Figure 2, A-D), and marked tubular atrophy was also found (Figure 2, B, D, and F). Tubular enlargement was associated with dilation of glomerular Bowman's space (Figure 2B). Most of the tissue 
sections showed important interstitial fibrosis localized predominantly in the peritubular area. Deposition of fibrotic material was more pronounced near the wall of small cysts. Focal cellular inflammatory infiltrates were also found (Figure 2, C-E), occasionally including fibroblasts (Figure 2E). Vascular sclerosis and lumen narrowing were consistently documented (Figure 2F). This pattern of structural changes consistently characterized all samples collected in the tissue areas between large cysts and belonging to the intermediate volume as identified at CT scan. The localization of important renal fibrosis in these samples was confirmed by Sirius Red staining of collagen deposition (Figure 3). Several glomeruli showed marked thickening of Bowman's capsule associated with concentric collagen deposition in periglomerular fibrotic areas (Figure 3, A and F). Marked peritubular collagen staining was found in all samples and involved both dilated and small atrophic tubules (Figure 3 $B, D$, and E). Sirius Red staining also localized in vascular wall confirming severe vascular fibrosclerosis (Figure $3, \mathrm{E}$ and F). Important fibrosis was present within the cyst wall. As shown in detail in Figure 4, A and B, organized fibrils of collagen characterize cyst wall. Immunohistochemical analysis of the cellular infiltrate revealed that $\mathrm{CD}^{+} 8^{+}$
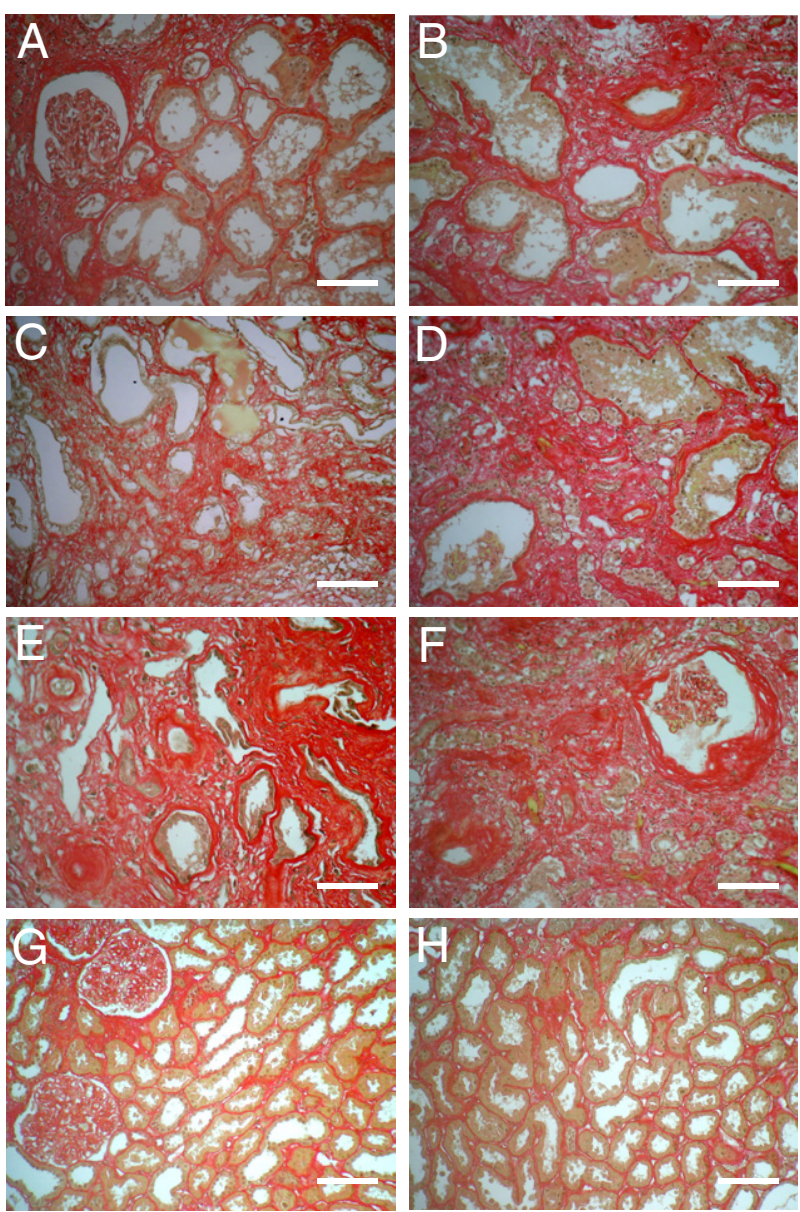

Figure 3. Representative images of collagen evaluation performed on kidney sections, in a patient with polycystic disease (A-F) and in a patient with normal-structure kidneys $(\mathbf{G}$ and $\mathbf{H})$, stained with Sirius Red. Scale $\mathrm{bar}=100 \mu \mathrm{m}$

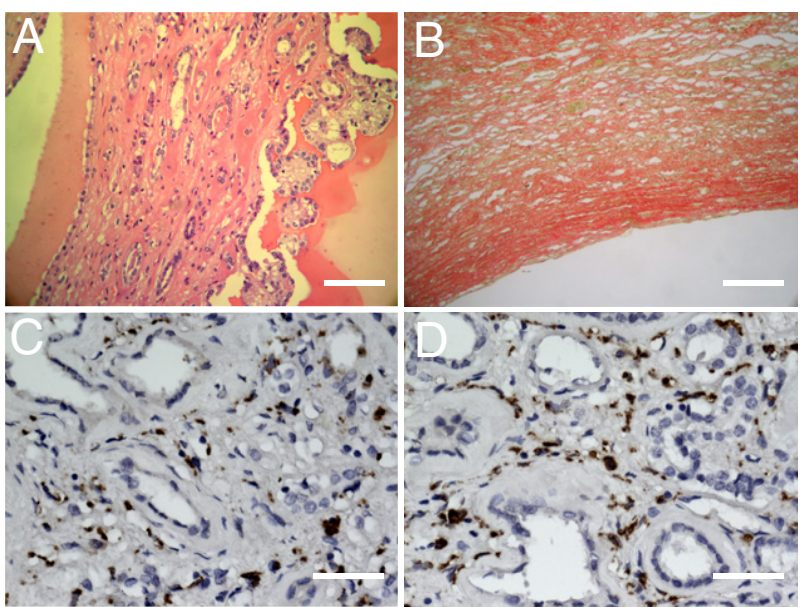

Figure 4. Representative immunohistochemical images of cellular infiltrates in kidney samples. Marked infiltration of $\mathrm{CD} 68^{+}$cells was observed in the intermediate volume, especially around atrophic tubuli and within fibrotic tissue. Scale bars: $100 \mu \mathrm{m}$ (A and $\mathbf{B}) ; 50 \mu \mathrm{m}(\mathbf{C}$ and $\mathbf{D})$.

monocytes were largely present in all biopsies. As shown in Figure 4, C and D, a marked infiltration of $\mathrm{CD}^{+}{ }^{+}$cells was observed in fibrotic intermediate renal volume and around atrophic tubules.

\section{Correlation between Intermediate Volume and Renal Function}

The analysis of the correlation between relative intermediate volume and GFR in the SIRENA cohort ${ }^{21}$ confirmed the significant correlation found in the original work ${ }^{15}$ involving the somatostatin cohort (SIRENA: $r=-0.69, P<$ 0.001 , regression line: $y=107.57-0.22 x$; somatostatin: $r=-0.79, P<0.005$, regression line: $y=106.30-$ $0.21 x$ ) with very similar correlation and slope. Again, no correlation was found between either total kidney or cyst volume and GFR (total kidney volume: SIRENA, $r=$ $-0.41, P=0.07$; somatostatin, $r=0.10, P=0.75$; total cyst volume: SIRENA, $r=-0.43, P=0.05$; somatostatin, $r=0.21, P=0.51$ ). As shown in Figure 5, we also found a significant correlation between relative intermediate volume and GFR in the pooled patient cohort $(r=-0.78$, $P<0.001$, regression line: $y=106.34-0.21 x)$. The regression slopes in the independent and pooled samples were very similar, despite differences in disease stage and CT acquisition protocol in the two cohorts. In the pooled patient cohort, absolute intermediate volume also significantly correlated with GFR $(r=-0.46, P<$ $0.01)$, but the correlation was weaker than for relative intermediate volume (Figure 6). No correlation was found between either total kidney or cyst volume and GFR. Residual parenchymal volume significantly correlated with renal function, although the strength of such correlation was low $(r=0.37, P<0.05)$.

\section{Prediction of GFR Decline by Intermediate Volume}

Out of the 13 ADPKD patients enrolled in the somatostatin cohort, ${ }^{22}$ one was excluded from the longitudinal analysis 


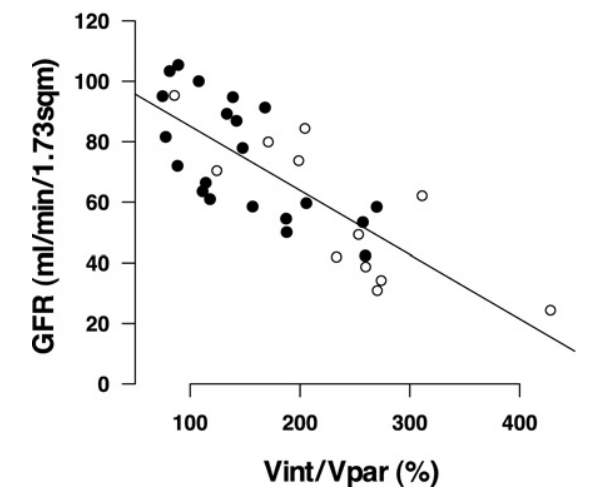

Figure 5. Correlation between relative intermediate volume [defined as the percentage of intermediate volume (Vint) over residual parenchymal volume (Vpar)] and glomerular filtration rate (GFR) in the combined SIRENA (closed circle $^{21}$ ) and somatostatin (open circles ${ }^{15}$ ) ADPKD cohorts. The correlation was found to be significant ( $\mathrm{r}=-0.78, P<0.001$ ), and the regression line fitted data from both patient cohorts (SIRENA: $r=-0.69$, $P<0.001$; somatostatin: $\mathrm{r}=-0.79, P<0.005)$, despite differences in CT acquisition protocol.

due to the limited follow-up (24 months), which led to an unreliable slope of GFR decline (GFR versus time: $r=$ $0.40, P=0.13)$, and another due to the advanced stage of renal disease at baseline, proximal to dialysis (eGFR = $24 \mathrm{~mL} /$ minute/1.73 $\mathrm{m}^{2}$ ), which led to low residual parenchymal volume (around $6 \%$ of the total kidney volume) and, consequently, a large intermediate/parenchymal volume ratio. In the remaining 11 patients, initial eGFR averaged $57 \pm 19 \mathrm{~mL} /$ minute $/ 1.73 \mathrm{~m}^{2}$ (range, 34 to 85 ). The mean observation period after first CT acquisition was $65 \pm 26$ months (range, 30 to 97). As expected, renal function progressively declined in all patients (Table 1), and the slope of eGFR ranged from -0.0790 to -0.5576
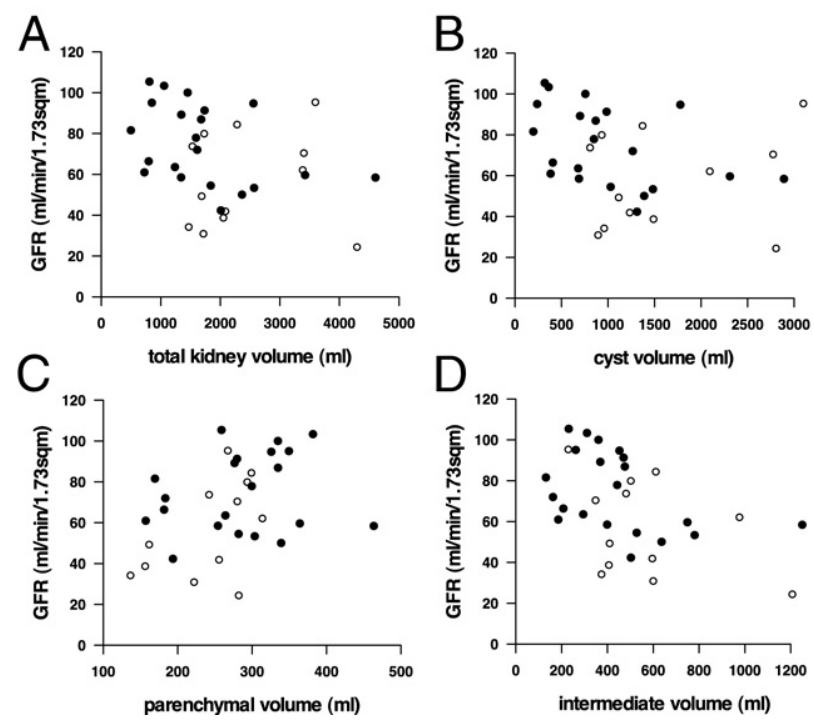

Figure 6. Correlation between individual tissue volumes and GFR in the combined SIRENA (closed circles ${ }^{21}$ ) and somatostatin (open circles ${ }^{15}$ ) ADPKD cohorts. No correlation was found between GFR and either total kidney ( $\mathrm{r}=$ $-0.31, P=0.081)(\mathbf{A})$ or cyst volume $(\mathrm{r}=-0.27, P=0.125)(\mathbf{B})$; residual parenchymal volume significantly correlated with renal function, although the strength of such correlation was low $(r=0.37, P<0.05)(\mathbf{C})$; absolute intermediate volume significantly correlated with GFR $(\mathrm{r}=-0.46, P<0.01)$ (D), but the correlation was much weaker than for the relative one.
Table 1. Individual Data Related to ADPKD Patients Included in the Investigation of Long-Term Prediction of Functional Loss by Intermediate Volume

\begin{tabular}{ccccc}
\hline $\begin{array}{r}\text { Patient } \\
\text { number }\end{array}$ & $\begin{array}{c}\text { eGFR } \\
\text { at CT }\end{array}$ & $\begin{array}{c}\text { Predialysis } \\
\text { follow-up } \\
\text { (months) }\end{array}$ & $\begin{array}{c}\text { eGFR } \\
\text { monthly } \\
\text { decline }\end{array}$ & $\begin{array}{c}\text { Vint/Npar } \\
(\%)\end{array}$ \\
\hline 1 & 42.7 & 30 & -0.5350 & 274 \\
2 & 73.4 & 95 & -0.3458 & 199 \\
3 & 62.0 & 81 & -0.3112 & 171 \\
4 & 34.1 & 53 & -0.4131 & 270 \\
5 & 38.3 & 33 & -0.3956 & 260 \\
6 & 74.2 & 36 & -0.5576 & 204 \\
7 & 37.8 & 97 & -0.1697 & 124 \\
8 & 55.2 & 74 & -0.3975 & 253 \\
9 & 42.1 & 45 & -0.5378 & 233 \\
10 & 84.8 & 86 & -0.0790 & 85 \\
11 & 81.0 & 87 & -0.4516 & 311 \\
\hline
\end{tabular}

Patients were enrolled in the previous CT-based somatostatin study. ${ }^{22}$ eGFR monthly decline was defined as the slope of eGFR regression ( $\mathrm{mL} /$ minute/1.73 $\mathrm{m}^{2} /$ month) versus time of observation.

eGFR, glomerular filtration rate estimated using plasma creatinine and the abbreviated MDRD formula $\left(\mathrm{mL} /\right.$ minute $\left./ 1.73 \mathrm{~m}^{2}\right)$; Vint, intermediate volume; Vpar, parenchymal volume.

$\mathrm{mL} /$ minute $/ 1.73 \mathrm{~m}^{2} /$ month. At CT scan, intermediate and parenchyma volumes were on average $503 \pm 197$ and $239 \pm 62 \mathrm{~mL}$, respectively. Intermediate volume relative to parenchymal volume ranged from $85 \%$ to $311 \%$ (mean $217 \% \pm 68 \%)$. Both absolute and relative intermediate volume (Figure 7A) at the time of first CT scan significantly correlated with the slope of eGFR decline (Vint, $r=$ $-0.63, P=0.037$; Vint/Vpar, $r=-0.79, P<0.004$; where Vint is the intermediate volume and Vpar is the parenchymal volume). Conversely, no significant negative correlation was found between either total kidney (Figure 7B) or cyst volume (Figure 7C) and long-term GFR decline. Baseline cyst volume showed a positive significant correlation with GFR decline, whereas total kidney volume showed no significant correlation. Additional analyses, performed including all 13 patients, confirmed the original results, showing that Vint/Vpar (\%) is significantly associated with slope of GFR decline $(r=-0.68$ and $P=$ 0.010 versus $r=-0.79, P<0.005$ ) whereas total kidney and cyst volumes are not (total kidney: $r=0.12, P=$ 0.698; cyst volume: $r=0.22, P=0.475)$.

\section{Discussion}

In the present study, we investigated the structural nature of what we have previously defined "intermediate" volume, radiologically identified as hypoenhanced regions of extracystic tissue in ADPKD kidneys on contrast-enhanced CT. Intermediate volume has specific image intensity on contrast-enhanced CT (ranging from 50 to 80 $\mathrm{HU}$ ), independently of patient size and of the amount of residual parenchyma. This, together with the availability of automatic algorithms for separating different tissue components, ensures the possibility to robustly and accurately identify it on CT scans. We found that histological samples of tissue corresponding to intermediate volume on CT images were consistently characterized by sparse dilated tubules, microcysts, and peritubular interstitial fi- 

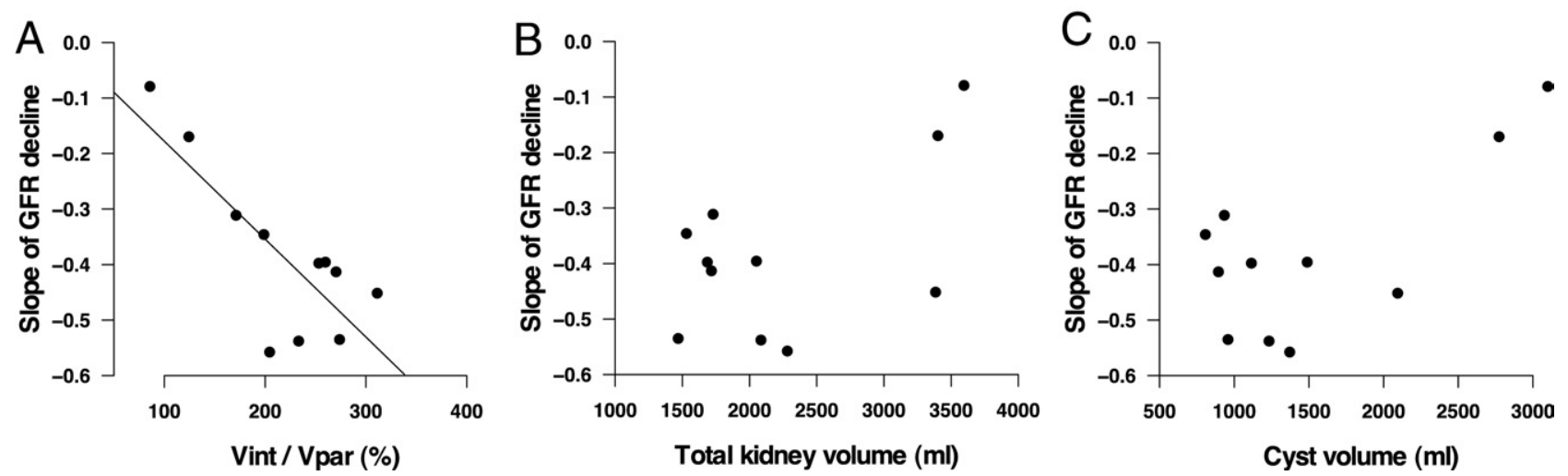

Figure 7. Correlation between relative intermediate volume, defined as percentage of intermediate volume (Vint) over residual parenchyma (Vpar) (A), total kidney $(\mathbf{B})$, and cyst volume $(\mathbf{C})$ and the slope of monthly GFR decline, determined based on the eGFR recorded during $7.3 \pm 2.8$ [3.0 to 10.4 ] years of clinical observation, in 11 of 13 ADPKD patients previously enrolled in the 6-month somatostatin study. ${ }^{22}$ The correlation between relative intermediate volume and GFR decline was found to be significant $(\mathrm{r}=-0.79, P=0.0038)$. No correlation was found between total kidney volume and GFR decline $(r=0.56, P=0.075)$, whereas a positive correlation, contrary to what was expected, was found between cyst volume and GFR decline $(r=0.69, P=0.019)$.

brosis, in line with previous histological findings showing the presence of interstitial fibrosis in ADPKD. ${ }^{20}$ From an imaging standpoint, hypoenhancement of intermediate volume on contrast-enhanced CT could then reflect the uptake of contrast agent in the sparse, dilated tubules and the reduced uptake throughout the fibrotic tissue, where peritubular capillaries are stretched with secondary tissue hypoperfusion, globally leading to a loss of enhancement on CT images at the macroscale.

Interstitial fibrosis, the main histological finding we documented in the samples harvested from tissue having a hypoenhanced tissue appearance on CT may derive from cortical ischemia as suggested by the severity of vascular lesions and lumen narrowing. Our results, showing marked infiltration of macrophages in the context of fibrotic tissue, suggest that the tubulointerstitial damage may be the consequence of cytokines/chemokines and growth factors release by inflammatory cells known to contribute to interstitial fibrosis. ${ }^{26}$ At histological examination, a peculiar finding was the prominent deposition of fibrotic material adjacent to the wall of small cysts as well as diffusely in the interstitium. Indeed, evidence is available that cyst-lining epithelial cells produce large amounts of structural (collagen I and III, laminin) ${ }^{27}$ and soluble extracellular matrix-associated proteins (TGF- $\beta$, periostin $)^{28}$ that accumulate around the cysts. Human and animal models of polycystic kidney disease also showed abnormal expression of matrix-degrading enzymes and inhibitors of metalloproteinases necessary for the remodeling of interstitial extracellular matrix. Together, these observations point to an important role of epithelial cells in the development of surrounding fibrosis in ADPKD. Abnormal expression of growth factors TGF- $\beta$ and PDGF by tubular epithelial cells may stimulate epithelial to mesenchymal transition and, ultimately, fibroblast generation in the cyst wall. ${ }^{26,29}$ This would account for the marked fibrosis of the cyst wall we found at histological examination of the removed ADPKD kidneys. Fibrosis within the cyst wall may be also induced by mechanical stimulation of cells due to hydrostatic pressure and development of organized structure of the wall to increase mechanical resistance. ${ }^{30}$ Tubular atrophy and interstitial fibrosis are known to occur concurrently to cyst development even in ADPKD patients and normal renal function, ${ }^{20}$ suggesting that characterization and monitoring of these phenomena could take place beginning in the earliest stages of the disease.

The compression of expanding cysts on residual parenchymal tissue and the stretching of kidney microvessels with secondary hypoperfusion ${ }^{31}$ have been suggested to play a role in the disruption of the normal tissue architecture that eventually sustain the progressive renal functional loss. In light of this hypothesis, the sequential measurement of total kidney and cyst volume has been put forth as a surrogate marker of disease progression, 9,11,13,32 and total kidney and cyst volume have been often adopted in clinical trials as outcome measures. Our group, among the others, recently found a significant effect of somatostatin and sirolimus on total kidney and cyst volume increase, ${ }^{15,21}$ further supporting the adoption of total and cyst volumes as outcome variables in the search for new therapies in the context of clinical studies.

Although it could be intuitively anticipated that some link between cyst burden and renal functional impairment exists, the relationship is not straightforward, as documented by the great diversity in total kidney and cyst volumes associated with any given eGFR in the ADPKD patient we studied. Moreover, evidence of slowing renal growth in the absence of a stabilizing effect in GFR decline in ADPKD patients receiving everolimus further highlights the limits of total kidney volume changes as surrogate marker of renal functional loss, ${ }^{33}$ and points out the need of novel biomarkers of functional loss, representative of disease progression. The loss of renal function in patients with ADPKD is insidious because it can remain undetected for several decades as a consequence of the ability of surviving nephrons to increase glomerular filtration rate in the face of the advancing renal structural damage. Tubulointerstitial disease is now recognized as an indispensable and prominent participant in the progression of renal disease. ${ }^{34}$ Landmark studies in the '60s were the first to highlight that the severity of renal fibrosis is the single best histological correlate of the decline in renal function and long-term prognosis. ${ }^{35,36}$ Thus, be- 
sides cyst enlargement and parenchymal compression, fibrotic transformation of the cyst wall and the adjacent interstitium in ADPKD may play a role in determining renal functional loss. Current findings that intermediate volume, as determined by CT, is mainly constituted by interstitial fibrosis allows one to consider this imaging parameter as a strong candidate marker for monitoring disease progression and to anticipate the long-term renal functional outcome in ADPKD patients.

Here, histological findings on the nature of intermediate volume have been coupled to further investigations on the relationships between intermediate volume and renal function. In an independent cohort of 21 ADPKD patients (SIRENA cohort), we found a strong correlation between intermediate volume relative to parenchyma and GFR measured at the time of CT imaging. Furthermore, in follow-up data on 13 patients, originally enrolled in the somatostatin study, with normal renal function or moderate renal dysfunction at the time of CT imaging, intermediate volume significantly correlated with the slope of GFR decline over the 3- to 8-year follow-up period.

These findings indicate that the most severe condition for a patient seems to be associated with the presence of large portions of fibrotic tissue relative to the preserved parenchyma and that this volume ratio is more readily related to both GFR and GFR decline than the absolute volumes alone. This is particularly relevant for the potential that intermediate volume may have for early stratification of ADPKD patients, in addition to monitoring disease progression. In fact, although GFR is a late marker of kidney damage in ADPKD, the possibility of stratifying patients according to their rate of disease progression based on an early image-based evaluation of relative intermediate volume opens new prognostic and monitoring perspectives, as well as new windows for evaluating early treatment strategies.

There are a number of limitations to be considered in the interpretation of these results. Present findings were obtained in a small number of patients and are therefore to be regarded as hypothesis generating, needing confirmation. The small number of patients may also explain the lack of a significant relation between total kidney or cyst volume and GFR, at variance to the findings of the CRISP (Consortium for Radiologic Imaging Studies of Polycystic Kidney Disease) study ${ }^{13}$; however, it should be noted that in that study, correlations were found to be significant on very large patients cohorts, suggesting that kidney and cyst volume are indeed weak indicators of loss of renal function in these patients. Another limitation is that the technique used to quantify intermediate volume is time consuming, and could probably not be directly applied in clinical practice as it is; however, it may be useful in clinical studies. It would be useful to develop both a clinically applicable tool for automatic image processing and a simpler CT-based score designed to grade fibrosis to potentially stratify patients according to their rate of disease progression. Histopathology was performed on three ADPKD patients with end stage renal disease. This should be considered as a limitation, however, since in these patients we found extensive kidney regions with a radiological appearance equivalent to that identified as intermediate in our previous studies, we assumed that such radiological pattern within the ADPKD kidney would be reflected by similar histopathological findings in other stages of the disease. This is consistent with a previous study ${ }^{20}$ showing the presence of fibrosis both in early and in late ADPKD stages. Finally, it has to be taken into account that use of contrast agent for CT renal imaging has a potential risk especially in patients with reduced GFR. ${ }^{37}$

In conclusion, we provided evidence that kidney tissue identified in ADPKD patients as intermediate on contrastenhanced CT mainly corresponds to regions of interstitial fibrosis at histological characterization and that intermediate volume is tightly connected to GFR and its longterm decline. As such, this parameter may represent a promising marker for early stratification of ADPKD patients, as well as for monitoring disease progression. Further studies on larger cohorts are now needed to establish intermediate volume as a biomarker of disease progression and interstitial fibrosis as a potential therapeutic target in ADPKD.

\section{Acknowledgments}

We thank Dr. Flavio Gaspari for renal functional evaluation using iohexol clearance and Dr. Irene Cattaneo for helping with the histological analyses.

\section{References}

1. Grantham JJ, Cowley BJ, Torres VE: Progression of autosomal dominant polycystic kidney disease (ADPKD) to renal failure. The Kidney: Physiology \& Pathophysiology. Edited by Seldin DW, Giebisch G. Philadelphia, Lippincott Williams \& Wilkins, 2000, pp 2513-2536

2. Gabow PA: Autosomal dominant polycystic kidney disease. N Engl J Med 1993, 329:332-342

3. Hateboer N, v Dijk MA, Bogdanova N, Coto E, Saggar-Malik AK, San Millan JL, Torra R, Breuning M, Ravine D: Comparison of phenotypes of polycystic kidney disease types 1 and 2. European PKD1-PKD2 Study Group. Lancet 1999, 353:103-107

4. Grantham JJ: Polycystic kidney disease: neoplasia in disguise. Am J Kidney Dis 1990, 15:110-116

5. Choukroun G, Itakura Y, Albouze G, Christophe JL, Man NK, Grunfeld $J P$, Jungers $P$ : Factors influencing progression of renal failure in autosomal dominant polycystic kidney disease. J Am Soc Nephrol 1995, 6:1634-1642

6. Grantham JJ: Mechanisms of progression in autosomal dominant polycystic kidney disease. Kidney Int Suppl 1997, 63:S93-S97

7. Gabow PA, Johnson AM, Kaehny WD, Kimberling WJ, Lezotte DC, Duley IT, Jones RH: Factors affecting the progression of renal disease in autosomal-dominant polycystic kidney disease. Kidney Int 1992, 41:1311-1319

8. Chapman AB: Cystic disease in women: clinical characteristics and medical management. Adv Ren Replace Ther 2003, 10:24-30

9. Grantham JJ, Chapman AB, Torres VE: Volume progression in autosomal dominant polycystic kidney disease: the major factor determining clinical outcomes. Clin J Am Soc Nephrol 2006, 1:148-157

10. Franz KA, Reubi FC: Rate of functional deterioration in polycystic kidney disease. Kidney Int 1983, 23:526-529

11. Grantham JJ, Torres VE, Chapman AB, Guay-Woodford LM, Bae KT, King BF Jr., Wetzel LH, Baumgarten DA, Kenney PJ, Harris PC, Klahr S, Bennett WM, Hirschman GN, Meyers CM, Zhang X, Zhu F, Miller JP: Volume progression in polycystic kidney disease. N Engl J Med 2006, 354:2122-2130 
12. Bae KT, Commean PK, Lee J: Volumetric measurement of renal cysts and parenchyma using MRI: phantoms and patients with polycystic kidney disease. J Comput Assist Tomogr 2000, 24:614-619

13. Chapman AB, Guay-Woodford LM, Grantham JJ, Torres VE, Bae KT, Baumgarten DA, Kenney PJ, King BF Jr., Glockner JF, Wetzel LH, Brummer ME, O'Neill WC, Robbin ML, Bennett WM, Klahr S, Hirschman GH, Kimmel PL, Thompson PA, Miller JP: Renal structure in early autosomal-dominant polycystic kidney disease (ADPKD): the Consortium for Radiologic Imaging Studies of Polycystic Kidney Disease (CRISP) cohort. Kidney Int 2003, 64:1035-1045

14. Bae KT, Zhu F, Chapman AB, Torres VE, Grantham JJ, Guay-Woodford LM, Baumgarten DA, King BF Jr., Wetzel LH, Kenney PJ, Brummer ME, Bennett WM, Klahr S, Meyers CM, Zhang X. Thompson PA, Miller JP: Magnetic resonance imaging evaluation of hepatic cysts in early autosomal-dominant polycystic kidney disease: the Consortium for Radiologic Imaging Studies of Polycystic Kidney Disease cohort. Clin J Am Soc Nephrol 2006, 1:64-69

15. Antiga L, Piccinelli M, Fasolini G, Ene-lordache B, Ondei P, Bruno S, Remuzzi G, Remuzzi A: Computed tomography evaluation of autosomal dominant polycystic kidney disease progression: a progress report. Clin J Am Soc Nephrol 2006, 1:754-760

16. Chapman $\mathrm{AB}$ : Improving clinical trial design for inquiries into the mechanisms of cyst growth in ADPKD. Kidney Int 2009, 75:139-141

17. Chang MY, Parker E, Ibrahim S, Shortland JR, Nahas ME, Haylor JL, Ong AC: Haploinsufficiency of $\mathrm{Pkd} 2$ is associated with increased tubular cell proliferation and interstitial fibrosis in two murine Pkd2 models. Nephrol Dial Transplant 2006, 21:2078-2084

18. Hassane S, Leonhard WN, van der Wal A, Hawinkels LJ, Lantinga-van Leeuwen IS, ten Dijke P, Breuning MH, de Heer E, Peters DJ: Elevated TGFbeta-Smad signalling in experimental Pkd1 models and human patients with polycystic kidney disease. J Pathol 2010, 222 21-31

19. Park EY, Seo MJ, Park JH: Effects of specific genes activating RAGE on polycystic kidney disease. Am J Nephrol 2010, 32:169-178

20. Zeier M, Fehrenbach P, Geberth S, Mohring K, Waldherr R, Ritz E: Renal histology in polycystic kidney disease with incipient and advanced renal failure. Kidney Int 1992, 42:1259-1265

21. Perico N, Antiga L, Caroli A, Ruggenenti P, Fasolini G, Cafaro M, Ondei P, Rubis N, Diadei O, Gherardi G, Prandini S, Panozo A, Bravo RF, Carminati S, De Leon FR, Gaspari F, Cortinovis M, Motterlini N, Ene-lordache B, Remuzzi A, Remuzzi G: Sirolimus therapy to halt the progression of ADPKD. J Am Soc Nephrol 2010, 21:1031-1040

22. Ruggenenti $P$, Remuzzi A, Ondei P, Fasolini G, Antiga L, Ene-lordache B, Remuzzi G, Epstein FH: Safety and efficacy of long-acting somatostatin treatment in autosomal-dominant polycystic kidney disease. Kidney Int 2005, 68:206-216
23. Zhang Y, Kong J, Deb DK, Chang A, Li YC: VitaminDreceptor attenuates renal fibrosis by suppressing the renin-angiotensin system. J Am Soc Nephrol, 21:966-973

24. Gaspari F, Perico N, Ruggenenti P, Mosconi L, Amuchastegui CS Guerini E, Daina E, Remuzzi G: Plasma clearance of nonradioactive iohexol as a measure of glomerular filtration rate. J Am Soc Nephrol 1995, 6:257-263

25. Levey AS, Greene T, Kusek J, Beck GJ, Group: for the MDRD Study A simplified equation to predict glomerular filtration rate from serum creatinine (abstract). J Am Soc Nephrol 2000, 11:155A

26. Perico N, Benigni A, Remuzzi G: Present and future drug treatments for chronic kidney diseases: evolving targets in renoprotection. Nat Rev Drug Discov 2008, 7:936-953

27. Joly D, Berissi S, Bertrand A, Strehl L, Patey N, Knebelmann B: Laminin 5 regulates polycystic kidney cell proliferation and cyst formation. J Biol Chem 2006, 281:29181-29189

28. Wallace DP, Quante MT, Reif GA, Nivens E, Ahmed F, Hempson SJ, Blanco G, Yamaguchi T: Periostin induces proliferation of human autosomal dominant polycystic kidney cells through alphaV-integrin receptor. Am J Physiol Renal Physiol 2008, 295:F1463-F1471

29. Wilson PD, Du J, Norman JT: Autocrine, endocrine and paracrine regulation of growth abnormalities in autosomal dominant polycystic kidney disease. Eur J Cell Biol 1993, 61:131-138

30. Gupta V, Grande-Allen KJ: Effects of static and cyclic loading in regulating extracellular matrix synthesis by cardiovascular cells. Cardiovasc Res 2006, 72:375-383

31. Chapman AB, Johnson A, Gabow PA, Schrier RW: The renin-angiotensin-aldosterone system and autosomal dominant polycystic kidney disease. N Engl J Med 1990, 323:1091-1096

32. Grantham JJ: Clinical practice. Autosomal dominant polycystic kidney disease. N Engl J Med 2008, 359:1477-1485

33. Walz G, Budde K, Mannaa M, Nurnberger J, Wanner C, Sommerer C Kunzendorf U, Banas B, Horl WH, Obermuller N, Arns W, Pavenstadt H, Gaedeke J, Buchert M, May C, Gschaidmeier H, Kramer S, Eckardt KU: Everolimus in patients with autosomal dominant polycystic kidney disease. N Engl J Med 2010, 363:830-840

34. Nath KA: Tubulointerstitial changes as a major determinant in the progression of renal damage. Am J Kidney Dis 1992, 20:1-17

35. Risdon RA, Sloper JC, De Wardener HE: Relationship between renal function and histological changes found in renal-biopsy specimens from patients with persistent glomerular nephritis. Lancet 1968, 2:363-366

36. Schainuck LI, Striker GE, Cutler RE, Benditt EP: Structural-functional correlations in renal disease. II. The correlations. Hum Pathol 1970 $1: 631-641$

37. Katzberg RW, Haller C: Contrast-induced nephrotoxicity: clinical landscape. Kidney Int 2006, 69:S3-S7 